hep-th/yymmnnn

HWM-07-2

EMPG-07-02

\title{
Worldsheet Instantons and a Null String Limit of Born-Infeld Theory
}

\author{
Gerald A. Goldin ${ }^{a}$, Nick E. Mavromatos ${ }^{b}$ and Richard J. Szabo ${ }^{c}$ \\ a Departments of Mathematics and Physics, Rutgers University, \\ Busch Campus, Piscataway, New Jersey 08854, USA \\ Email: geraldgoldin@dimacs.rutgers.edu \\ ${ }^{b}$ Department of Physics, King's College London, \\ Strand, London WC2R 2LS, UK \\ Email: nikolaos.mavromatos@kcl.ac.uk \\ ${ }^{c}$ Department of Mathematics and Maxwell Institute for Mathematical Sciences, \\ Heriot-Watt University, Colin Maclaurin Building, \\ Riccarton, Edinburgh EH14 $4 A S, U K$ \\ Email: R.J.Szabo@ma.hw.ac.uk
}

\begin{abstract}
For a superstring theory in four spacetime dimensions, we propose a modification of the Born-Infeld action that possesses a well-defined tensionless limit. We interpret this as describing the effective target space dynamics of null strings on a D3-brane. We argue that such a modification can be induced by nonperturbative contributions from instantons in the worldsheet $\sigma$-model describing string propagation on the brane.
\end{abstract}


In this paper, we consider an abelian Born-Infeld theory of four-dimensional superstrings on spacetime-filling D3-branes. We demonstrate that the usual Born-Infeld action has a consistent modification that possesses a well-defined null string (i.e., tensionless) limit, for appropriate brane worldvolumes. The suggested form of the modified action is analogous to an earlier proposal [1] for modifying Born-Infeld electromagnetism to permit a consistent Galilean $(c \rightarrow \infty)$ limit. In the present, fully relativistic context, we argue that such a modification may be induced by nonperturbative effects on the null string worldsheet, in the form of worldsheet instantons. Our analysis provides an independent indication that nonperturbative worldsheet effects must be included in order to give a target space interpretation to $\sigma$-models for null strings propagating in gauge backgrounds. We also discuss how our results can be extended to nonabelian Born-Infeld theory describing the target space dynamics of coincident multiple D-branes.

Study of the tensionless limit of strings and $p$-branes spans three decades. The original concept of the null string is due to Schild [2], who gave a classical worldsheet action in the limit where the Regge slope $\alpha^{\prime}$ of the string becomes infinite. Subsequently it was found that upon quantising the null string worldsheet field theory, there appears to be no critical dimension of spacetime [3], and that this result is consistent with conformal invariance. However, the conclusion is still a matter of debate [4]. It has been pointed out that the issue depends crucially on the way in which one quantises the theory [5]. Physically, the results can be interpreted as follows. The specific operator ordering for $p=1$ is associated with viewing the null string as a collection of particles moving under certain conditions, where the absence of a critical dimension is expected on physical grounds. But if one ignores the particle interpretation, and restricts attention only to the appropriate tensionless limit of the conformal anomaly, then nontrivial central charge terms appear-leading, in that case, to a critical dimension. However, it has been argued even here that the limit in which the string tension $T \rightarrow 0$ can be taken in a way that leads to a null string conformal algebra with a vanishing critical dimension [6]. These ambiguities all can be traced back to operator orderings. By carrying out BRST quantisation for a model of $p$-branes with second class constraints, it was shown in [5] that for $p>1$, the operator orderings which induce the critical dimension in the $p=1$ case are forbidden, and thus impose no restriction on the dimensionality of the target space.

The zero-tension limit of strings and D-branes attracted renewed attention in [7], for both physical and mathematical reasons. New worldsheet actions and consistent quantisation schemes have been proposed for tensionless strings [8]. They have been argued to be instrumental in a variety of applications such as the AdS/CFT correspondence [9] and the twistor string formulation of supersymmetric Yang-Mills theory [10]. The tensionless limit of gauged, non-compact Wess-Zumino-Witten (WZW) models, which arises when the level $k$ of the underlying Kac-Moody algebra with a formally divergent central charge assumes a critical value (equal to the dual Coxeter number of the gauge group), has been analysed in [11, 12]. One finds that the central charge of all higher spin generators is fixed to a critical value which is not seen by the usual Virasoro symmetry. From a physical point of view, such limiting cases of WZW models might describe a topological phase of high energy quantum chromodynamics [13]. While the results of [12] do not seem to support this idea, it was pointed out in 13 , that nonperturbative worldsheet configurations - such as instantons [14] - play a crucial role in consistently yielding the above limit, as well as in the breaking of the topological symmetry. Such configurations were ignored in [12], which might 
account for the authors' unexpected results concerning the decoupling of worldsheet gravity (the Liouville mode).

Here we study the tensionless limit of strings and $p$-branes from an alternative (but equally problematic) point of view to the worldsheet and worldvolume perspectives described above - that of the target space dynamics of background gauge fields in which open strings propagate. It is well known that for oriented open strings of coupling $g_{s} \ll 1$, and finite tension

$$
T:=\frac{1}{2 \pi \alpha^{\prime}} \neq 0,
$$

propagating in weak abelian gauge field backgrounds, the bosonic part of the low-energy effective target space dynamics is described by the Born-Infeld action [15]-17,

$$
S_{\mathrm{BI}}^{(p+1)}=\frac{T^{(p+1) / 2}}{g_{s}} \int \mathrm{d}^{p+1} X \sqrt{-\operatorname{det}_{p+1}\left(G_{\mu \nu}+T^{-1} F_{\mu \nu}\right)},
$$

where the inverse power of the string coupling indicates that this term arises from the lowest order disk diagram in open string perturbation theory. The gauge fields $A_{\mu}, \mu=0,1, \ldots, p$, with field strength $F_{\mu \nu}$, are assumed to be living on the longitudinal $p+1$ directions of a $\mathrm{D} p$-brane, which from a worldsheet perspective are characterised by $\sigma$-model fields $X^{\mu}$ with Neumann boundary conditions at the boundary of the worldsheet disk. We allow for gravitational backgrounds, so that $G_{\mu \nu}$ is an arbitrary metric on the brane, but we set the axion, dilaton and $B$-field to zero for simplicity. If there is a critical target space dimension $d^{*}$, then the remaining $d^{*}-p-1$ string embedding fields $X^{i}$ have fixed Dirichlet boundary conditions. If there is no critical dimension, then one may consider four-dimensional superstrings on spacetime-filling D3-branes. This is the case to which, for definiteness, we now restrict ourselves. For the remainder of this paper, we also set the string coupling $g_{s}=1$.

Now four-dimensional abelian Born-Infeld actions are particularly easy to manipulate, as they can be expressed in terms of two geometrical invariants [16] corresponding to the Yang-Mills and instanton densities. These may be written,

$$
I_{1}=\frac{1}{2} G^{\mu \lambda} G^{\nu \rho} F_{\mu \nu} F_{\lambda \rho}, \quad I_{2}=-\frac{1}{4} \epsilon^{\mu \nu \lambda \rho} F_{\mu \nu} F_{\lambda \rho} .
$$

Using the identity

$$
\operatorname{det}_{4}\left(G_{\mu \nu}+T^{-1} F_{\mu \nu}\right)=\operatorname{det}(G)\left(1+T^{-2} I_{1}-T^{-4} I_{2}^{2}\right)
$$

which is particular to the case of four spacetime dimensions, we can express the Born-Infeld action (2) in the form

$$
S_{\mathrm{BI}}^{(4)}=\int \mathrm{d}^{4} X \sqrt{-\operatorname{det}(G)} \mathcal{R}
$$

where

$$
\mathcal{R}=\sqrt{T^{4}+T^{2} I_{1}-I_{2}^{2}}
$$

Note that with our choice of units, the string tension $T$ is playing the role of the critical Born-Infeld electric field strength; i.e., the maximum electric field intensity that can be accommodated by a real-valued Born-Infeld Lagrangian (6). In the tensionless limit $\alpha^{\prime} \rightarrow \infty$, the abelian Born-Infeld action (5) becomes imaginary, and is thus undefined. This instability reflects the physical fact that in this limit, there is no string tension to hold the strings together in any background electric field [17]. 
There is an analogy between this situation and the situation discussed in [1, where the Galilean limit $c \rightarrow \infty$ of Born-Infeld theory was considered. In that limit, the standard Born-Infeld action vanishes. This prompted the authors of [1] to propose an example of how to modify the Born-Infeld action, so as to obtain a nontrivial action and nontrivial, nonlinear constitutive equations for electromagnetism (or, analogously, for Yang-Mills theory) in the Galilean limit. Of course, for strings there is no Galilean limit per se; but one can nevertheless follow the procedure in [1] of introducing terms that result in a well-defined Born-Infeld action in the null string limit. Thus motivated, we propose the analogous modification of the abelian Born-Infeld Lagrangian (6), given by,

$$
\mathcal{R}^{\prime}=\sqrt{\left(T^{4}+\lambda_{2}^{4}\right)+\left(T^{2}+\lambda_{1}^{2}\right) I_{1}-I_{2}^{2}}
$$

where $\lambda_{1}, \lambda_{2}$ are appropriate dimensionful constants, dependent on $\alpha^{\prime}$, but taking finite values $\hat{\lambda}_{1}, \hat{\lambda}_{2}$ respectively in the limit $\alpha^{\prime} \rightarrow \infty$. We describe their form and origin in more detail below. Notice that we have modified the constant term inside the square root of the BornInfeld Lagrangian, which otherwise tends to zero in the $\alpha^{\prime} \rightarrow \infty$ limit. In that limit, the modified Born-Infeld action is now given by,

$$
\mathcal{R}_{\infty}^{\prime}:=\lim _{\alpha^{\prime} \rightarrow \infty} \mathcal{R}^{\prime}=\sqrt{\hat{\lambda}_{2}^{4}+\hat{\lambda}_{1}^{2} I_{1}-I_{2}^{2}}
$$

The constitutive equations

$$
\mathcal{G}^{\mu \nu}:=-\frac{1}{2} \frac{\partial \mathcal{R}}{\partial F_{\mu \nu}}=-G^{\mu \lambda} G^{\nu \rho} F_{\lambda \rho} \frac{\partial \mathcal{R}}{\partial I_{1}}-\frac{1}{4} \epsilon^{\mu \nu \lambda \rho} F_{\lambda \rho} \frac{\partial \mathcal{R}}{\partial I_{2}}
$$

lead to the nonlinear electromagnetic equations of motion $\nabla_{\mu} \mathcal{G}^{\mu \nu}=0$, and they have welldefined nonzero limits given by

$$
\frac{\partial \mathcal{R}_{\infty}^{\prime}}{\partial I_{1}}=\frac{\hat{\lambda}_{1}^{2}}{2 \mathcal{R}_{\infty}^{\prime}}, \quad \frac{\partial \mathcal{R}_{\infty}^{\prime}}{\partial I_{2}}=-\frac{I_{2}}{\mathcal{R}_{\infty}^{\prime}} .
$$

Notice that the invariant structure of the modified Lagrangian is identical to that of the original one, and it thus possesses the same worldvolume symmetries. Requiring positivity of the argument of the square root in (8) imposes restrictions on the relative strengths of the various terms. For relatively weak background gauge fields, the Born-Infeld Lagrangian is real and defined; hence the modification (77)-(8) is the analog of the Galilean limit modification of the standard Born-Infeld action of [1]. This procedure is somewhat reminescent of the approach of [18], which shows how to obtain a well-defined Born-Infeld action in the limit where the brane tension vanishes. The modification in this case is interpreted physically as the splitting of the D-brane worldvolume into a collection of tensile strings.

Next we relate this target space modification of the Born-Infeld action to the worldsheet dynamics of the null string. The form of the parameters $\lambda_{j}$ can be constrained by the requirement that they do not affect perturbative string scattering amplitudes. The latter are expressed as series in powers of $\sqrt{\alpha^{\prime}} p$, where $p$ is a characteristic momentum scale of the low energy string excitations. This implies that the $\lambda_{j}$, as functions of $T$, should vanish faster than the inverse of any polynomial in $T$ as $T \rightarrow \infty$; i.e., $\lambda_{j}$ approaches 0 faster than any power of the worldsheet $\sigma$-model coupling constant $\sqrt{\alpha^{\prime}}$ approaches 0 ; so that the 
perturbation expansion remains intact to all orders. For example, the functional dependence of the $\lambda_{j}$ on $T$ may take the form

$$
\lambda_{j}=\hat{\lambda}_{j} \mathrm{e}^{-T / \hat{\lambda}_{j}}
$$

Then we may also propose that (11) holds in the nonperturbative regime $\alpha^{\prime} \rightarrow \infty$. The issue now is how such $1 / \alpha^{\prime}$ corrections can arise at the level of the worldsheet theory. We argue that they do so through worldsheet instantons [19], which are already known [13, 14] to play a role in the tensionless limit of strings represented by two-dimensional $\sigma$-models.

In fact, the worldsheet $\sigma$-model action describing open string dynamics on the D3-brane is given by

$$
S_{\sigma}[X]=\frac{T}{2} \int \mathrm{d}^{2} z G_{i \bar{i}}\left(\bar{\partial} X^{i} \partial X^{\bar{i}}+\partial X^{i} \bar{\partial} X^{\bar{i}}\right)+\oint \mathrm{d} \tau A_{\mu} \partial_{\tau} X^{\mu}
$$

Here the worldsheet is a disk, whose bulk can be regarded as a sphere $\mathbb{C P}^{1} \cong \mathbb{C} \cup\{\infty\}$, with local complex coordinates $z, \bar{z}$; and whose boundary is a circle $\mathbb{S}^{1}$ with coordinate $\tau$. We have assumed that the brane worldvolume is a Kähler manifold, with Euclidean complex coordinates $X^{i}, X^{\bar{i}}, i=1,2$, in order to ensure formal convergence of the $\sigma$-model path integral

$$
\mathcal{Z}=\int \mathrm{D} X \mathrm{e}^{-S_{\sigma}[X]}
$$

where the integration is over string maps from the disk to the four-dimensional target space. (Analytic continuation to Minkowski time should be done at the end.) Since the D-brane in this instance carries a $\operatorname{spin}^{c}$ structure, the global Freed-Witten anomalies cancel in (13) [20]. The metric $G_{i \bar{i}}(X)$ associated with the image $X$ of a spherical worldsheet is expressed in terms of the Kähler potential $\mathcal{K}(X)$ as

$$
G_{i \bar{i}}(X)=\partial_{i} \partial_{\bar{i}} \mathcal{K}(X) .
$$

To ensure conformal invariance, we further require that the spacetime metric $G_{i \bar{i}}$ be Ricci flat. Then there are no harmonic $(0,2)$ or $(2,0)$ forms. The Kähler form $\omega_{i \bar{i}}=-\omega_{\bar{i} i}=$ i $G_{i \bar{i}}$ is a harmonic $(1,1)$ form with respect to the metric $G$.

In the traditional analysis the null string amplitudes vanish, because in an effective action approach they appear as expansion terms of the form $\left(\alpha^{\prime}\right)^{n}\left\langle V_{i_{1}} \cdots V_{i_{n}}\right\rangle$, where the $V_{i_{k}}$ are appropriate vertex operators, and where the amplitude is of the order $\partial^{2 n}$ in a derivative expansion. Thus when $\alpha^{\prime} \rightarrow \infty$, one has $\left\langle V_{i_{1}} \cdots V_{i_{n}}\right\rangle \rightarrow 0$, and all local correlation functions are trivial. This means that the worldsheet field theory defined by (12)-(13) becomes essentially a two-dimensional topological $\sigma$-model. This topological field theory localizes onto its instanton sectors. The instantons are holomorphic functions of the worldsheet coordinates,

$$
\frac{\partial X^{i}}{\partial \bar{z}}=0
$$

which implies that only one of the two terms in the bulk action of (12) will contribute. The path integral (13) then reduces to an integral over the finite dimensional instanton moduli space. The worldsheet zero mode integration yields the Born-Infeld action, while the integration over nontrivial instanton sectors will contribute the above nonperturbative $\mathrm{e}^{-1 / \alpha^{\prime}}$ 
corrections to the null string amplitudes to all orders. These open string instantons can be described as holomorphic maps from $\mathbb{C P}^{1}$ to the brane worldvolume (i.e. as holomorphic curves of genus zero with possible self-intersections), with fixed monodromies around nontrivial one-cycles in the spacetime prescribed by the gauge field background $A_{\mu}$.

For a holomorphic instanton $X_{n}$ wrapping $n$ times around a single embedded $\mathbb{C P}^{1}$, the bulk contribution to the action is

$$
S_{n}:=S_{\sigma}\left[X_{n}\right]=n T,
$$

while the contribution to the path integral from winding the disk boundary $w$ times around a single embedded $\mathbb{S}^{1}$ is $\mathcal{W}^{w}$, where $\mathcal{W}$ is the Wilson line associated to the background $A_{\mu}$. As these latter contributions have no effect on the $\alpha^{\prime} \rightarrow \infty$ limit of the theory, we henceforth focus on only the bulk contributions. As we now demonstrate, the presence of these instantons cures the instability of ordinary Born-Infeld theory in the null string limit. At the quantum level, they act to suppress the spontaneous creation of charged null open strings from the vacuum [17, 21].

To illustrate, we describe explicitly the case where the four-dimensional background spacetime is the complex projective plane $\mathbb{C P}^{2}$. The qualitative results which follow, however, hold for any Ricci-flat Kähler four-manifold with second Betti number $b_{2}=1$, and can be generalized easily to the cases of multiple two-cycles by considering each type of instanton in turn. In this case, the background geometry (14) is provided by the Fubini-Study metric, which can be computed from the Kähler potential

$$
\mathcal{K}(X)=\log \left(1+X^{i} X^{\bar{i}}\right) .
$$

The construction of worldsheet instantons $X_{n}: \mathbb{C P}^{1} \rightarrow \mathbb{C P}^{2}$ proceeds as follows [22]. Any such holomorphic map may be defined by setting

$$
X_{n}(z)=\left[p_{0}(z), p_{1}(z), p_{2}(z)\right],
$$

where $z \in \mathbb{C}$, the triple $\left[u_{0}, u_{1}, u_{2}\right]$ denotes homogeneous coordinates on $\mathbb{C P}^{2}$, and the $p_{j}$ are polynomials with no common zeroes. Then the topological degree of $X_{n}$ is the integer

$$
d=\max _{j=0,1,2}\left\{\operatorname{deg} p_{j}(z)\right\},
$$

so that $n=n(d, r):=d-r-2$ by the Riemann-Hurwitz theorem, where $r$ is the total ramification index of $X_{n}$ with $0 \leq r \leq \frac{3}{2} d-3$. The canonical example of such an instanton is provided by the holomorphic map $X_{n}(z)=\left[1,(z+1)^{d-r+1}, z^{d}\right]$.

The localization of the $\sigma$-model partition function (13) in the tensionless limit $T \rightarrow 0$ onto an integral over the finite dimensional instanton moduli space is in this case given by

$$
\mathcal{Z}_{\infty}:=\lim _{\alpha^{\prime} \rightarrow \infty} \mathcal{Z}=\sum_{d \in \mathbb{Z}} \sum_{r=0}^{\frac{3}{2}|d|-3} \int_{\mathcal{M}_{d, r}} \mathrm{~d} \mu(m) \mathrm{e}^{-S_{n(|d|, r)}}
$$

where $\mathcal{M}_{d, r}$ is the stratified moduli space of holomorphic maps $\mathbb{C P}^{1} \rightarrow \mathbb{C P}^{2}$ of degree $d$ and ramification index $r$, and the moduli $m$ can be determined from the independent polynomial coefficients in (18). $\mathcal{M}_{d, r}$ is naturally a connected complex manifold of complex dimension 
$3 d-r+2$ [22. Unfortunately, beyond these facts the geometry of these moduli spaces is not generally known (see [22] for some low degree examples), and so it is not possible to further specify the moduli space integration in (20). Therefore, in the following we will work in a fixed instanton background, and drop the integral over moduli in (20). This is like a dilute instanton-gas approximation, where interactions between instantons are ignored. It will turn out to be the correct prescription for obtaining the string version of the $\alpha^{\prime} \rightarrow \infty$ limit discussed in the context of the Lagrangian (8).

We denote by $\langle\langle\mathcal{O}(X)\rangle\rangle:=\int \mathrm{D} X^{\prime} \mathrm{e}^{-S_{\sigma}\left[X^{\prime}\right]} \mathcal{O}(X) / \int \mathrm{d} X_{0} \mathrm{e}^{-S_{\sigma}\left[X_{0}\right]}$ the average of any operator $\mathcal{O}(X)$ in the $\sigma$-model over the non-zero modes $X^{\prime}$ with respect to the instanton action (12), normalized by the zero-instanton partition function. Ideally, we would like to calculate the average $\langle\langle\mathcal{R}\rangle\rangle$ of the Born-Infeld operator (66) in a fixed gauge field background, but in general an expansion of the square root in unmanageable. Instead we work in the fixed instanton background just discussed, and calculate

$$
\left\langle\left\langle\mathcal{R}^{2}\right\rangle\right\rangle=T^{4}\langle\langle 1\rangle\rangle+T^{2}\left\langle\left\langle G^{\mu \lambda}(X) G^{\nu \rho}(X)\right\rangle\right\rangle F_{\mu \nu} F_{\lambda \rho}-I_{2}^{2} .
$$

Note that the expectation value of the topological invariant $I_{2}^{2}$ is trivial, because it does not depend on the background geometry. Each average in (21) induces a sum over terms $\mathrm{e}^{-n T}$ with the appropriate nonperturbative structures. In the limit $T \rightarrow 0$, only instantons of very large degree $n \rightarrow \infty$ will contribute, yielding the desired modification (8) of the Born-Infeld Lagrangian.

In the fixed instanton background, one may replace the averages in (21) by quantum mechanical expectation values with respect to a state $\left|\psi_{n}\right\rangle$ corresponding to a specific instanton number $n \in \mathbb{Z}$; i.e., $\langle\langle\mathcal{O}\rangle\rangle:=\left\langle\psi_{n}|\mathcal{O}| \psi_{n}\right\rangle$. Here $n<0$ correspond to the contributions from anti-holomorphic maps, and in the generalization to spaces with second Betti number $b_{2}>1, n$ would label an integer $b_{2}$-vector representing multiple instanton contributions. These states define a complete orthonormal system of vectors in the quantum Hilbert space of the $\sigma$-model, $\left\langle\psi_{n} \mid \psi_{m}\right\rangle=\delta_{n m}$, which decomposes the Hilbert space into superselection sectors. One has $X\left|\psi_{n}\right\rangle=X_{n}\left|\psi_{n}\right\rangle$, where $X_{n}$ denotes the specified instanton background such as (18). Inserting the sum over such a complete set of states into (21), the off-diagonal matrix elements of $\mathcal{R}$ vanish as they correspond to instanton modes belonging to distinct superselection sectors, and we obtain

$$
\left\langle\psi_{n}\left|\mathcal{R}^{2}\right| \psi_{n}\right\rangle=\sum_{m \in \mathbb{Z}}\left\langle\psi_{n}|\mathcal{R}| \psi_{m}\right\rangle\left\langle\psi_{m}|\mathcal{R}| \psi_{n}\right\rangle=\left\langle\psi_{n}|\mathcal{R}| \psi_{n}\right\rangle^{2}
$$

We may therefore write the instanton-averaged Born-Infeld Lagrangian in closed form, as

$$
\langle\langle\mathcal{R}\rangle\rangle=\sqrt{T^{4}\langle\langle 1\rangle\rangle+T^{2}\left\langle\left\langle G^{\mu \lambda}(X) G^{\nu \rho}(X)\right\rangle\right\rangle F_{\mu \nu} F_{\lambda \rho}-I_{2}^{2}} .
$$

This leads to the identification of the parameters $\lambda_{j}$ in (8) with expressions taking the form of (11).

It should be stressed, however, that (11) is merely the simplest form that consistent nonperturbative expressions in $\alpha^{\prime}$ might take, and that a more precise calculation of the above instanton contributions (including the moduli space integrations) should be expected to lead to more complicated functions. It would be interesting to carry out an analysis in this context along the lines of [19], who show that worldsheet instanton contributions to 
tree-level target space scattering amplitudes renormalize the spacetime superpotential and can destabilize vacuum configurations by inducing tadpole graphs for massless particles. Nevertheless, it is encouraging that the rough computation of worldsheet instanton effects outlined above leads to this form.

We conclude by indicating briefly how our analysis can be generalized to nonabelian Born-Infeld theory. For nonabelian gauge fields, the action (22) is modified by including a symmetrized trace operation (over the group indices), denoted STr, acting on the square root [16]. One still has formally the four-dimensional determinant identity (4) for nonabelian field strengths. But the resulting Lagrangian is no longer expressed in terms of just two geometrical invariants, because $\operatorname{STr}(\mathcal{R})$ includes all possible orderings of the $F_{\mu \nu}$ factors. The formal way to proceed is first to expand the abelian Born-Infeld Lagrangian (66) in (even) powers of $F$ to define spacetime tensors $\mathcal{C}^{\mu_{1} \nu_{1} \cdots \mu_{2 k} \nu_{2 k}}$, through the formula

$$
\mathcal{R}=\sum_{k=1}^{\infty} \mathcal{C}^{\mu_{1} \nu_{1} \cdots \mu_{2 k} \nu_{2 k}} F_{\mu_{1} \nu_{1}} \cdots F_{\mu_{2 k} \nu_{2 k}}
$$

Writing $F_{\mu \nu}=F_{\mu \nu}^{a} T_{a}$ with $T_{a}$ the orthonormal generators of the gauge group in the fundamental representation, the nonabelian Born-Infeld Lagrangian is then given by

$$
\operatorname{STr}(\mathcal{R})=\sum_{k=1}^{\infty} d_{a_{1} \cdots a_{2 k}} \mathcal{C}^{\mu_{1} \nu_{1} \cdots \mu_{2 k} \nu_{2 k}} F_{\mu_{1} \nu_{1}}^{a_{1}} \cdots F_{\mu_{2 k} \nu_{2 k}}^{a_{2 k}},
$$

where the totally symmetric tensors $d_{a_{1} \cdots a_{l}}:=\operatorname{STr}\left(T_{a_{1}} \cdots T_{a_{l}}\right)$ are the invariant tensors for the adjoint action of the corresponding Lie algebra.

For the gauge group $S U(2)$, one can explicitly sum the series (25) [16]. In this case the generators satisfy $T_{a} T_{b}=\delta_{a b}+\mathrm{i} \epsilon_{a b c} T_{c}$, and so $d_{a_{1} \cdots a_{2 k}}=2 \delta_{\left\{a_{1} a_{2}\right.} \cdots \delta_{\left.a_{2 k-1} a_{2 k}\right\}}$. The simple structure of the invariant tensors enables one to write the $S U(2)$ Lagrangian in terms of three geometric invariants, $\operatorname{Tr} I_{1},\left(\operatorname{Tr} I_{2}\right)^{2}$, and $\operatorname{Tr} I_{2}^{2}$, as

$$
\operatorname{STr}\left(\mathcal{R}_{S U(2)}\right)=\sqrt{T^{4}+T^{2} \operatorname{Tr} I_{1}-\frac{1}{3}\left(\left(\operatorname{Tr} I_{2}\right)^{2}+2 \operatorname{Tr} I_{2}^{2}\right)} .
$$

In the abelian case the latter two invariants in (26) coincide, and the Lagrangian reduces to (6). Again in direct analogy with the development in [1, we have in place of (7) the modification of the nonabelian Born-Infeld action given by

$$
\operatorname{STr}\left(\mathcal{R}_{S U(2)}^{\prime}\right)=\sqrt{\left(T^{4}+\lambda_{2}^{4}\right)+\left(T^{2}+\lambda_{1}^{2}\right) \operatorname{Tr} I_{1}-\frac{1}{3}\left(\left(\operatorname{Tr} I_{2}\right)^{2}+2 \operatorname{Tr} I_{2}^{2}\right)},
$$

which has a well-defined null string limit with the same geometric invariant structure. In particular, the nonabelian constitutive equations

$$
\mathcal{G}_{a}^{\mu \nu}:=-\frac{1}{2} \frac{\partial \mathrm{S} \operatorname{Tr}(\mathcal{R})}{\partial F_{\mu \nu}^{a}}
$$

and the resulting equations of motion again have well-defined limits as $\alpha^{\prime} \rightarrow \infty$. It is clear in this case that precisely the same worldsheet instanton mechanism as for the abelian case gives rise to the nonperturbative parameters $\lambda_{j}$ in the modified Lagrangian (27). It would be interesting to extend this analysis to higher rank gauge groups. In general there are only 
partial results describing the invariant tensors $d_{a_{1} \cdots a_{2 k}}$, and the Lagrangians will generically be functions of many more geometric invariants. It would also be interesting to generalize the above results to higher dimensional manifolds, describing string propagation in general $\mathrm{D} p$-branes. In [23] it is shown how Dirac-Born-Infeld Lagrangians can be written as square roots of quadratic forms in any dimension.

Acknowledgments. N.M. wishes to thank I. Bakas, and G.G. wishes to thank V. Shtelen, for discussions. G.G. acknowledges the support of the Leverhulme Trust (U.K.) for a Visiting Professorship at King's College London during his 2004-2005 sabbatical leave, when this research originated. The work of N.M. was supported in part through the Marie Curie Research and Training Network Universenet (MRTN-CT-2006-035863). The work of R.S. was supported in part by the Marie Curie Research and Training Network ForcesUniverse (MRTN-CT-2004-005104).

\section{References}

[1] G. A. Goldin and V. M. Shtelen, "Generalizations of Yang-Mills Theory with Nonlinear Constitutive Equations," J. Phys. A 37, 10711 (2004) arXiv:hep-th/0401093.

[2] A. Schild, "Classical Null Strings," Phys. Rev. D 16, 1722 (1977).

[3] F. Lizzi, B. Rai, G. Sparano and A. Srivastava, "Quantization of the Null String and Absence of Critical Dimensions," Phys. Lett. B 182, 326 (1986); A. Karlhede and U. Lindstrom, "The Classical Bosonic String in the Zero Tension Limit," Class. Quant. Grav. 3, L73 (1986); R. Amorim and J. Barcelos-Neto, "Strings with Zero Tension," Z. Phys. C 38, 643 (1988); J. Barcelos-Neto and M. Ruiz-Altaba, "Superstrings with Zero Tension," Phys. Lett. B 228, 193 (1989); I. A. Bandos and A. A. Zheltukhin, "Hamiltonian Mechanics and Absence of Critical Dimensions for Null Membranes," Sov. J. Nucl. Phys. 50, 556 (1989) [Yad. Fiz. 50, 893 (1989)]; H. J. de Vega, I. Giannakis and A. Nicolaidis, "String Quantization in Curved Spacetimes: Null String Approach," Mod. Phys. Lett. A 10, 2479 (1995) [arXiv:hep-th/9412081].

[4] J. Gamboa, C. Ramirez and M. Ruiz-Altaba, "Quantum Null (Super)Strings," Phys. Lett. B 225, 335 (1989).

[5] P. Bozhilov, "Tensionless Branes and the Null String Critical Dimension," Mod. Phys. Lett. A 13, 2571 (1998) arXiv:hep-th/9711136.

[6] F. Lizzi, "The Zero Tension Limit of the Virasoro Algebra and the Central Extension," Mod. Phys. Lett. A 9, 1495 (1994) arXiv:hep-th/9404148]; A. Nicolaidis, J. E. Paschalis and P. I. Porfyriadis, "String Tension and the Generation of the Conformal Anomaly," Phys. Rev. D 58, 047901 (1998) [arXiv:hep-th/9702185].

[7] H. J. De Vega and A. Nicolaidis, "Strings in Strong Gravitational Fields," Phys. Lett. B 295, 214 (1992); O. J. Ganor and A. Hanany, "Small $E_{8}$ Instantons and Tensionless Noncritical Strings," Nucl. Phys. B 474, 122 (1996) [arXiv:hep-th/9602120]; C. O. Lousto and N. Sanchez, "String Dynamics in Cosmological and Black Hole Backgrounds: 
The Null String Expansion," Phys. Rev. D 54, 6399 (1996) arXiv:gr-qc/9605015; O. J. Ganor, "Six-Dimensional Tensionless Strings in the Large $N$ Limit," Nucl. Phys. B 489, 95 (1997) arXiv:hep-th/9605201]; A. Hanany and I. R. Klebanov, "On Tensionless Strings in 3+1 Dimensions," Nucl. Phys. B 482, 105 (1996) arXiv:hep-th/9606136; P. Mayr, "Mirror Symmetry, $\mathcal{N}=1$ Superpotentials and Tensionless Strings on CalabiYau Fourfolds," Nucl. Phys. B 494, 489 (1997) arXiv:hep-th/9610162.

[8] G. Savvidy, "Conformal Invariant Tensionless Strings," Phys. Lett. B 552, 72 (2003); "Tensionless Strings: Physical Fock Space and Higher Spin Fields," Int. J. Mod. Phys. A 19, 3171 (2004) |arXiv:hep-th/0310085|; I. Antoniadis and G. Savvidy, "Physical Fock Space of Tensionless Strings," arXiv:hep-th/0402077; "Tensionless Strings, Correspondence with $S O(D, D)$ Sigma-Model," Phys. Lett. B 615, 285 (2005) arXiv:hep-th/0502114.

[9] D. Mateos, T. Mateos and P. K. Townsend, "Supersymmetry of Tensionless Rotating Strings in $\mathrm{AdS}_{5} \times \mathbb{S}^{5}$ and Nearly-BPS Operators," JHEP 0312, 017 (2003) arXiv:hep-th/0309114; A. Sagnotti and M. Tsulaia, "On Higher Spins and the Tensionless Limit of String Theory," Nucl. Phys. B 682, 83 (2004) |arXiv:hep-th/0311257); A. Bredthauer, U. Lindström, J. Persson and L. Wulff, "Type IIB Tensionless Superstrings in a pp-Wave Background," JHEP 0402, 051 (2004) arXiv:hep-th/0401159; G. Bonelli, "On the Boundary Gauge Dual of Closed Tensionless Free Strings in AdS," JHEP 0411, 059 (2004) arXiv:hep-th/0407144].

[10] W. Siegel, "Untwisting the Twistor Superstring," arXiv:hep-th/0404255; I. A. Bandos, J. A. de Azcarraga and C. Miquel-Espanya, "Superspace Formulations of the (Super)Twistor String," JHEP 0607, 005 (2006) arXiv:hep-th/0604037.

[11] U. Lindstrom and M. Zabzine, "Tensionless Strings, WZW Models at Critical Level and Massless Higher Spin Fields," Phys. Lett. B 584, 178 (2004) arXiv:hep-th/0305098.

[12] I. Bakas and C. Sourdis, "On the Tensionless Limit of Gauged WZW Models," JHEP 0406, 049 (2004) arXiv:hep-th/0403165]; "Aspects of WZW Models at Critical Level," Fortsch. Phys. 53, 409 (2005) [arXiv:hep-th/0501127].

[13] J. R. Ellis and N. E. Mavromatos, "High Energy QCD as a Topological Field Theory," Eur. Phys. J. C 8, 91 (1999) arXiv:hep-ph/9807451].

[14] A. V. Yung, "The Broken Phase of the Topological $\sigma$-Model," Int. J. Mod. Phys. A 10, 1553 (1995) arXiv:hep-th/9401124]; "Instanton Dynamics in the Broken Phase of the Topological $\sigma$-Model," Int. J. Mod. Phys. A 11, 951 (1996) arXiv:hep-th/9502149 ; "Worldsheet Instantons in the 2D Black Hole," Int. J. Mod. Phys. A 9, 591 (1994).

[15] E. S. Fradkin and A. A. Tseytlin, "Nonlinear Electrodynamics from Quantized Strings," Phys. Lett. B 163, 123 (1985); "Quantum String Theory Effective Action," Nucl. Phys. B 261, 1 (1985).

[16] A. A. Tseytlin, "Born-Infeld Action, Supersymmetry and String Theory," in: The Many Faces of the Superworld, ed. M. A. Shifman (World Scientific, Singapore, 2000), p. 417 arXiv:hep-th/9908105]. 
[17] J. Ambjørn, Y. M. Makeenko, G. W. Semenoff and R. J. Szabo, "String Theory in Electromagnetic Fields," JHEP 0302, 026 (2003) arXiv:hep-th/0012092.

[18] U. Lindstrom and R. von Unge, "A Picture of D-Branes at Strong Coupling," Phys. Lett. B 403, 233 (1997) arXiv:hep-th/9704051]; H. Gustafsson and U. Lindstrom, "A Picture of D-Branes at Strong Coupling II: Spinning Partons," Phys. Lett. B 440, 43 (1998) arXiv:hep-th/9807064.

[19] M. Dine, N. Seiberg, X.-G. Wen and E. Witten, "Nonperturbative Effects on the String Worldsheet," Nucl. Phys. B 278, 769 (1986); "Nonperturbative Effects on the String Worldsheet. 2," Nucl. Phys. B 289, 319 (1987).

[20] D. S. Freed and E. Witten, "Anomalies in String Theory with D-Branes," Asian J. Math. 3, 819 (1999) arXiv:hep-th/9907189].

[21] C. Bachas and M. Porrati, "Pair Creation of Open Strings in an Electric Field," Phys. Lett. B 296, 77 (1992) arXiv:hep-th/9209032.

[22] L. Lemaire and J. C. Wood, "On the Space of Harmonic 2-Spheres in $\mathbb{C P}^{2}$," Internat. J. Math. 7, 211 (1996) arXiv:dg-ga/9510003 ; T. A. Crawford, "The Space of Harmonic Maps from the 2-Sphere to the Complex Projective Plane," Canad. Math. Bull. 40, 285 (1997) arXiv:dg-ga/9512004.

[23] D. B. Fairlie, "Dirac-Born-Infeld Equations," Phys. Lett. B 456, 141 (1999) arXiv:hep-th/9902204]; "Lagrange Brackets and $U(1)$ Fields," Phys. Lett. B 484, 333 (2000) arXiv:hep-th/0005009. 